\title{
Do tumor location and grade affect survival in pT2N0M0 esophageal squamous cell carcinoma?
}

Dongrong Situ, MD, Junye Wang, MD, PhD, Peng Lin, MD, PhD, Hao Long, MD, PhD, Lanjun Zhang, MD, $\mathrm{PhD}$, Tiehua Rong, $\mathrm{MD}$, and Guowei $\mathrm{Ma}, \mathrm{MD}, \mathrm{PhD}$

Background: The TNM staging system for esophageal cancer in the seventh edition of the AJCC Cancer Staging Manual incorporates tumor grade and location for staging pT2-3N0M0 esophageal squamous cell carcinoma. Patients with pT2NOM0, classified as stage IIA according to the sixth edition of the AJCC Cancer Staging Manual, can now be classified as stage IB, IIA, or IIB. We discuss whether these changes lead to a better prediction of the prognosis of these patients and aimed to find out other factors to forecast patient prognosis.

Methods: We retrospectively analyzed 317 patients with postoperative pathologic stage T2N0M0 who underwent esophagectomy between 1990 and 2005 at Sun Yat-sen University Cancer Center. We performed univariate and multivariate analyses to identify prognostic factors for survival and used the Kaplan-Meier method to demonstrate the prognostic efficacy of each prognostic factor, including tumor grade and location.

Results: The 5-year overall survival was $57 \%$, with a median survival of 84.5 months (6.94 years). Univariate analysis indicated that age, alcohol consumption, and tumor grade were associated with survival. Multivariate Cox proportional hazard regression analysis revealed that alcohol consumption and tumor grade were independent prognostic factors. Survival analysis using the Kaplan-Meier method demonstrated age, cigarette smoking, alcohol consumption, tumor grade, and location as prognostic factors.

Conclusions: For pT2N0M0 esophageal squamous cell carcinoma, the seventh edition of the AJCC Cancer Staging Manual does not provide a more distinguishable prediction of prognosis compared with the sixth edition. Tumor grade is an independent prognostic factor in patients with pT2N0M0 esophageal squamous cell carcinoma, whereas tumor location is not. Furthermore, alcohol consumption is an independent prognostic factor that may imply a worse prognosis. (J Thorac Cardiovasc Surg 2013;146:45-51)

Esophageal squamous cell carcinoma (ESCC) has a poor prognosis and is one of the most common and aggressive cancers found throughout Asia, especially in China. ${ }^{1,2}$ Surgery is the main treatment, but the overall survival remains unsatisfactory. Half of those patients had potentially curative resections with a 3.5-year survival of $41.3 \%$; for those resected for palliation, the 3.5 -year survival was $7.3 \%$. The overall survival remains at $20 \%$ to $25 \%$. $^{3}$

Accurate cancer staging is important for formulation of treatment protocols, therapeutic evaluations, and assessment of prognosis. In addition, it allows for treatment comparisons between different centers. The seventh edition of

\footnotetext{
From the Cancer Center, Sun Yat-sen University State Key Laboratory of Oncology in South China, Guangzhou, China.

This work was supported by the Fundamental Research Funds for the Central Universities (No. 09YKPY51); the Science and Technology Planning Project of Guangdong Province, China (No. 2010B31500010); and the National High Technology Research and Development Program of China (863 Program; No. 2009AA02Z421)

Disclosures: Authors have nothing to disclose with regard to commercial support. Received for publication May 30, 2012; revisions received Oct 23, 2012; accepted for publication Jan 21, 2013; available ahead of print March 13, 2013.

Address for reprints: Guowei Ma, MD, PhD, The Department of Thoracic Surgery Cancer Center, Sun Yat-sen University State Key Laboratory of Oncology in South China, 651 Dongfengdong Rd, Guangzhou, China (E-mail: gzlmst@yeah.net). $0022-5223 / \$ 36.00$

Copyright (c) 2013 by The American Association for Thoracic Surgery http://dx.doi.org/10.1016/j.jtcvs.2013.01.034
}

the AJCC Cancer Staging Manual was released in 2010, ${ }^{4}$ updating the TNM staging system for esophageal cancer. In this manual, tumor location is simplified and includes the esophagogastric junction and proximal $5 \mathrm{~cm}$ of the stomach. Furthermore, $\mathrm{T}$ classifications are redefined and T4 is subclassified. Regional lymph nodes are redefined, and $\mathrm{N}$ is subclassified according to the number of regional metastatic lymph nodes. $\mathrm{M}$ is redefined, and stages are separated for squamous cell carcinoma and adenocarcinoma. Stages are reassigned using the $\mathrm{T}, \mathrm{N}, \mathrm{M}$, and $\mathrm{G}$ classifications. $^{4}$

T2NOM0 ESCC was previously classified as stage IIA according to the sixth edition of the AJCC Cancer Staging Manual, ${ }^{5}$ but it can now be classified as stage IB, IIA, and IIB according to the seventh edition. We retrospectively analyzed 317 patients with T2NOM0 ESCC who underwent esophagectomy to investigate whether the new stage classification meant varied prognosis and whether any other factors affected survival.

\section{PATIENTS AND METHODS \\ Patient Data Acquisition}

This study was approved by the Sun Yat-sen University Cancer Center Hospital Ethics Committee. All patients who underwent esophagectomy for esophageal cancer at the Thoracic Surgery Department of Sun Yat-sun University Cancer Center from 1990 to 2005 were reviewed. Among 


\section{Abbreviation and Acronym \\ $\mathrm{ESCC}=$ esophageal squamous cell carcinoma}

them, 317 who had pT2N0M0 ESCC and underwent curative (R0) resection were included in this study. All tumors were located in the thoracic segments, and no patient received neoadjuvant chemotherapy or radiotherapy.

\section{Clinical Data}

The study included 317 patients with T2NOM0 ESCC (222 male and 95 female) and a median age of 56 years (mean age, 55.6 years; standard deviation, 9.774) who underwent esophagectomy between 1990 and 2005. Of these, 186 patients $(58.7 \%)$ smoked cigarettes and 60 patients $(18.9 \%$ ) consumed alcohol (a drinker refers to those who drink $>100 \mathrm{~g} / \mathrm{d}$ for $>1$ year.). In regard to tumor location, $40(12.7 \%)$ were in the upper thoracic region, $218(69.0 \%)$ were in the middle thoracic region, and 58 $(18.4 \%)$ were in the lower thoracic region. According to tumor grade, $121(38.3 \%)$ were well differentiated (G1), $126(39.9 \%)$ were moderately differentiated (G2), and $69(21.8 \%)$ were poorly differentiated (G3). The 1 -, 3-, and 5-year overall survivals were $75 \%, 63 \%$, and $57 \%$, respectively, with a median survival of 84.5 months (6.94 years). Patient characteristics are summarized in Table 1.

\section{Tumor Location}

In this study, the medical records were accomplished before the publication of the seventh edition of the AJCC Cancer Staging Manual, ${ }^{4}$ so the relationship between the exact location of tumor and the new landmarks was not recorded. However, the distance from the tumor to the superior incisor has been measured by endoscopy and recorded in detail, and thus can be used to estimate the location of tumor. A tumor 15 to $20 \mathrm{~cm}$ away from the superior incisor was considered as cervical, whereas tumors 20 to $25 \mathrm{~cm}, 25$ to $30 \mathrm{~cm}$, and 30 to $40 \mathrm{~cm}$ were considered upper thoracic, middle thoracic, and lower thoracic, respectively.

\section{Statistical Analysis}

All potential and reported prognostic factors, including demographic data, cigarette smoking, and alcohol consumption; operative information; and tumor characteristics were analyzed. Patient data were evaluated according to pathologic stage classification of the sixth edition of the AJCC Cancer Staging Manual. ${ }^{5}$ Cervical, upper thoracic, middle thoracic, and lower thoracic were coded as 1,2,3, and 4, respectively, when tumor location was involved in the analysis. In terms of differentiation grade, tumors that were highly, intermediately, and poorly differentiated were coded as 1,2 , and 3 , respectively.

\section{Follow-up}

Postoperative patients were followed regularly at our outpatient department every 3 months in the first 2 years after operation, every 6 months in the third year, and once per year in the following 2 years. Outpatient department visits encompassed history taking, physical examination, chest x-ray, barium meal, abdominal type B ultrasonography, and tumor biomarkers, including squamous cell carcinoma and carcinoembryonic antigen. Endoscopic examination and computed tomography were carried out on occasion. Patients with survival time more than 5 years were contacted by our follow-up department via telephone or mail. Overall survival was defined as the time from operation to death or the last follow-up. The last follow-up checkpoint was April 2010. Fourteen of 317 patients were lost to follow-up.

\section{Statistics}

Overall survival was calculated on the basis of survival time from operation to death or the last follow-up. Life table analysis was used to calculate the 1-, 3-, and 5-year overall survival. Univariate analysis and multivariate Cox proportional hazard regression analysis were used to identify potential prognostic factors. Potential prognostic factors associated with survival probability that had a significance level of $P$ less than .10 were considered in multivariable Cox proportional hazard regression analysis. Patient survival curves were constructed using the Kaplan-Meier method. The log-rank test was used to compare survival differences between groups for each variable. All statistical calculations were performed using the Statistical Package for the Social Sciences version 17.0 (IBM SPSS Inc, Chicago, Ill).

\section{RESULTS}

\section{New Criteria for Staging}

The 317 patients with pT2NOM0 ESCC were divided into 3 subgroups, namely, IB, IIA, and IIB, according to the seventh edition of the AJCC Cancer Staging Manual. ${ }^{4}$ KaplanMeier analysis revealed no significant differences between the survival curves for each group. $P$ value calculated by the log-rank test was .305 , which indicated that the 3 subgroups resulting from the new criteria did not have a significantly different prognosis. The seventh edition did not provide a more distinguishable prediction of patients with pT2NOM0 ESCC (Figure 1).

\section{Prognostic Factors}

Prognostic factors affecting long-term survival are evaluated in Tables 2 and 3. Univariate analysis indicated that age $(P=.038)$, alcohol consumption $(P=.035)$, and tumor grade $(P=.007)$ were significantly associated with patient survival. Likewise, multivariate Cox proportional hazard regression analysis indicated that alcohol consumption $(P=.015)$ and tumor grade $(P=.011)$ were significantly associated with patient survival (Table 3).

The Kaplan-Meier method and log-rank test were used to construct survival curves for age, alcohol consumption, and cigarette smoking. The cutoff point for age was defined at the median age of 56 years. However, age as a continuous variable was not dichotomized elsewhere. Age $(P=.029)$, alcohol consumption $(P=.028)$, and cigarette smoking $(P=.039)$ were observed to be significantly associated with patient survival (Table 3). Furthermore, advanced age, cigarette smoking, and alcohol consumption were associated with worse prognosis (Figure 2).

\section{Role of Tumor Grade and Location in Esophageal Squamous Cell Carcinoma}

Univariate analysis and multivariable Cox proportional hazard regression analysis indicated that tumor grade was associated with patient survival $(P=.007$ and $P=.011$, respectively) (Tables 1 and 2). We also established the survival curves using the Kaplan-Meier method, which indicated that well-differentiated tumors had better prognosis (Figure 3). For tumor location, to exclude the effect of tumor grade, we also established the survival curves of different tumor location in each grade. For moderately and 
TABLE 1. Baseline characteristics of patients

\begin{tabular}{lc}
\hline \multicolumn{1}{c}{ Variables } & No. $(\%)$ or median \\
\hline Age, $y$ & 56 \\
Gender & \\
Male & $222(70.0 \%)$ \\
Female & $95(30.0 \%)$ \\
Cigarette smoking & \\
Yes & $186(58.7 \%)$ \\
No & $131(41.3 \%)$ \\
Alcohol consumption & \\
Yes & $60(18.9 \%)$ \\
No & $257(81.1 \%)$ \\
Family history of cancer & \\
Yes & $26(8.2 \%)$ \\
No & $291(91.8 \%)$ \\
Surgical approach & \\
Left thoracic & $249(78.5 \%)$ \\
Right thoracic & $68(21.5 \%)$ \\
Tumor location & \\
Upper thoracic & $40(12.7 \%)$ \\
Middle thoracic & $218(69.0 \%)$ \\
Lower thoracic & $58(18.4 \%)$ \\
Tumor grade & \\
Well differentiated $(\mathrm{G} 1)$ & $121(38.3 \%)$ \\
Moderately differentiated $(\mathrm{G} 2)$ & $126(39.9 \%)$ \\
Poorly differentiated $(\mathrm{G} 3)$ & $69(21.8 \%)$ \\
\hline
\end{tabular}

poorly differentiated pT2NOM0 ESCC, tumor location was not associated with patient survival $(P=.714$ and $P=.227$, respectively) (Figure 4$)$. For the well-differentiated tumor,

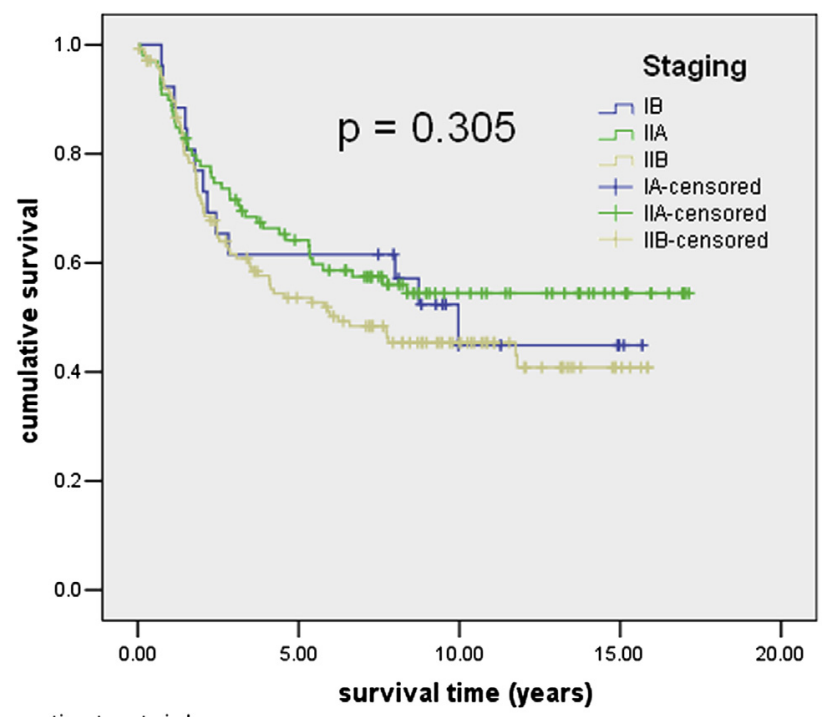

patients at risk

$\begin{array}{ccccc}\text { IB } & 26 & 12 & 3.5 & 1 \\ \text { IA } & 96 & 45.5 & 18 & 5.5 \\ \text { IIB } & 131.5 & 52 & 19 & 2.5\end{array}$

FIGURE 1. Kaplan-Meier survival curves for patients staged by the seventh edition of the AJCC Cancer Staging Manual. ${ }^{4}$ Survival differences were analyzed using Cox regression analysis.
TABLE 2. Possible prognostic factors and relative risks

\begin{tabular}{lccc}
\hline \multicolumn{1}{c}{ Possible prognostic factor } & $\boldsymbol{P}$ & HR & $\mathbf{9 5 \%}$ CI \\
\hline Gender & .606 & 1.187 & $0.619-2.273$ \\
Age & .038 & 1.023 & $1.001-1.045$ \\
Cigarette smoking & .263 & 1.360 & $0.794-2.329$ \\
Alcohol consumption & .035 & 1.570 & $1.032-2.389$ \\
Family history of cancer & .343 & 0.716 & $0.359-1.428$ \\
VC & .389 & 0.732 & $0.361-1.487$ \\
MVV & .267 & 1.009 & $0.993-1.026$ \\
FEV1 & .956 & 1.024 & $0.434-2.420$ \\
FEV1\% & .619 & 1.007 & $0.979-1.035$ \\
Blood loss & .725 & 1.000 & $0.999-1.001$ \\
3-FL & .223 & 0.647 & $0.322-1.302$ \\
Length of tumor & .372 & 1.043 & $0.951-1.143$ \\
Depth of tumor invasion & .516 & 1.131 & $0.780-1.639$ \\
Tumor grade & .007 & 1.373 & $1.091-1.728$ \\
Tumor location & .463 & 0.881 & $0.628-1.235$ \\
Postoperative anastomotic leakage & .379 & 1.381 & $0.673-2.835$ \\
Surgical approach & .215 & 1.461 & $0.802-2.661$ \\
No. of removed LNs & .938 & 0.999 & $0.969-1.030$ \\
\hline
\end{tabular}

$H R$, Hazard ratio; $C I$, confidence interval; $V C$, vital capacity; $M V V$, maximal voluntary ventilation; FEVI, forced expiratory volume in 1 second; $F E V I \%$, forced expiratory volume in $1 \mathrm{~s}$ expressed as percent predicted; 3-FL, 3-field lymphadenectomy; $L N$, lymph node.

tumor location was linked with patient survival $(P=.006)$ (Figure 4). Although the Kaplan-Meier method showed that tumor location was significantly associated with patient survival $(P=.015)$, it was not identified as an independent prognostic factor in multivariable Cox proportional hazard regression analysis.

\section{DISCUSSION}

The seventh edition of the AJCC Cancer Staging Man$u a l^{4}$ adopts evidence from evidence-based medicine and a new statistical model. For this revision, inputs from Asian scholars were invited because Asian countries have a higher incidence of ESCC, accounting for one third of patients with ESCC. The seventh edition incorporates tumor grade and location; however, these factors are less significant than TNM, which generally affects only the stage classification of patients with pT2-3NOM0. The new staging system specifically defines anatomic segmentation of esophageal cancer, considering the survival influence of organs adjacent to the tumors.

In the seventh edition of the AJCC Cancer Staging Man$u a l,{ }^{4}$ tumor location is redefined as the length between the upper incisor and the upper edge of the tumor, not the tumor center. The esophagus is now divided into cervical, upper thoracic, middle thoracic, and lower thoracic regions by 3 new landmarks, namely, the thoracic entrance, inferior border of azygos vein arch, and inferior border of inferior pulmonary vein.

In this study, the 5-year overall survival was $57 \%$, which was in accordance with the data previously reported. 
TABLE 3. Survival differences after multivariate Cox proportional hazard regression analysis

\begin{tabular}{|c|c|c|c|c|c|}
\hline Demographics & Patient, no. & Univariate log-rank $P$ & Multivariate regression $P$ & HR & $95 \% \mathrm{CI}$ \\
\hline Alcohol consumption & & .028 & .015 & 1.627 & $1.101-2.405$ \\
\hline Yes & 62 & & & & \\
\hline No & 240 & & & & \\
\hline Age (y) & & .029 & & & \\
\hline$<56$ & 60 & & & & \\
\hline$\geq 56$ & 238 & & & & \\
\hline Cigarette smoking & & .039 & & & \\
\hline Yes & 186 & & & & \\
\hline No & 131 & & & & \\
\hline Tumor location & & .015 & & & \\
\hline Upper thoracic & 40 & & & & \\
\hline Middle thoracic & 218 & & & & \\
\hline Lower thoracic & 58 & & & & \\
\hline Tumor grade & & .015 & .011 & 1.323 & $1.066-1.641$ \\
\hline Well differentiated (G1) & 121 & & & & \\
\hline Moderately differentiated (G2) & 126 & & & & \\
\hline Poorly differentiated (G3) & 69 & & & & \\
\hline
\end{tabular}

$H R$, Hazard ratio; $C I$, confidence interval.

Killinger and colleagues ${ }^{6}$ reported their experience with pathologic T2N0M0 disease treated using esophagectomy alone. In this retrospective analysis, the survival of patients with pT2NOM0 disease was on par with that of patients with pT1N0M0 ( $\sim 50 \%$ at 5 years; $P=.83)$ and trended toward improved survival over patients with pT3N0M0 disease $(P=.06)$. Tachibana and colleagues ${ }^{7}$ published the results of their study on patients with pT1 and pT2 ESCC who underwent esophagectomy. Among patients who were node negative, those with pT1 and pT2 tumors had similar cancer-specific survival. Rice and colleagues ${ }^{8}$ reported that 31 patients with pT2NOM0 ESCC who underwent surgery first had a 5-year overall survival of $61 \% \pm 9.3 \%$.

We performed a survival analysis for 317 patients with pT2NOM0 ESCC. Univariate analysis showed that age, alcohol consumption, and tumor grade were associated with patient survival. Furthermore, multivariable Cox proportional hazard regression analysis revealed that alcohol consumption and tumor grade were independent prognostic factors. Cigarette smoking was found to be associated with patient survival using the Kaplan-Meier method.

The prognostic role of age has been demonstrated in many previous studies. It has been confirmed that advanced age is associated with worse prognosis. Elsayed and colleagues ${ }^{9}$ reported that increasing age was a significant risk factor for mortality and survival after esophagectomy. The mortality was particularly high when associated with a preoperative cardiac or respiratory morbidity. Eloubeidi and colleagues ${ }^{10}$ showed that across all stages, age was an important predictor of survival in patients with esophageal carcinoma.

In regard to alcohol consumption, the predictive role on survival varies among studies. Kountourakis and colleagues $^{11}$ demonstrated that alcohol consumption influences the long-term outcome of patients with cT2NOM0 esophageal cancer, although different opinions exist. ${ }^{12}$ In our study, alcohol consumption was found to be strongly associated with poor survival.

Because cigarette smoking and alcohol consumption synergistically contribute to ESCC development, ${ }^{13-15}$ we evaluated smoking as a prognostic factor. Survival curves demonstrated that smoking is not only a risk factor for ESCC development but also a prognostic factor in patients already diagnosed with ESCC. Identical results were reported by Khan and colleagues ${ }^{16}$ in a cohort from the Kashmir Valley, India. Kountourakis and colleagues ${ }^{11}$ showed that smoking influenced long-term outcome in patients with cT2NOM0 esophageal cancer, a conclusion confirmed by Shitara and colleagues. ${ }^{17}$

Univariate analysis, multivariate Cox proportional hazard regression analysis, and the Kaplan-Meier method showed that tumor grade had a significant influence on survival of patients with pT2NOMO ESCC and was an independent prognostic factor, whereas tumor location was not.

Tumor grade was shown to be a prognostic indicator in perijunctional esophagogastric carcinoma long before the adoption of the new staging system ${ }^{18}$ and was incorporated in the new staging system ${ }^{19}$; however, its role in ESCC remains unclear. ${ }^{10,20,21}$ In a 292-patient study, $\mathrm{Li}$ and colleagues $^{2}$ showed that tumor grade was a prognostic factor in univariate analysis, but not in multivariate Cox regression analysis. Roder and colleagues ${ }^{22}$ reported that in squamous cell carcinoma-predominant databases, tumor differentiation was not significantly associated with survival.

Similar conflicting results have been obtained for tumor location. ${ }^{2,10,20}$ Although studies have shown that tumors in the lower segment of esophagus had better prognosis because of the increased sufficiency of resection, these 


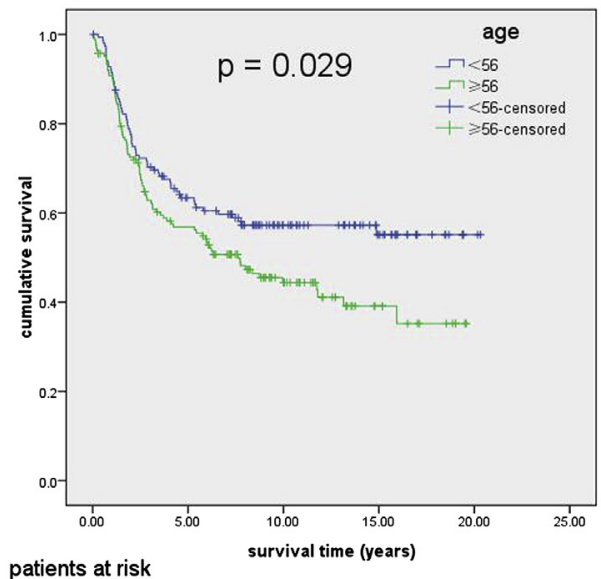

$\begin{array}{llllll}<56 & 147 & 71 & 36 & 13 & 1\end{array}$

$A \geqslant 56 \quad 158 \quad 70 \quad 27 \quad 7$

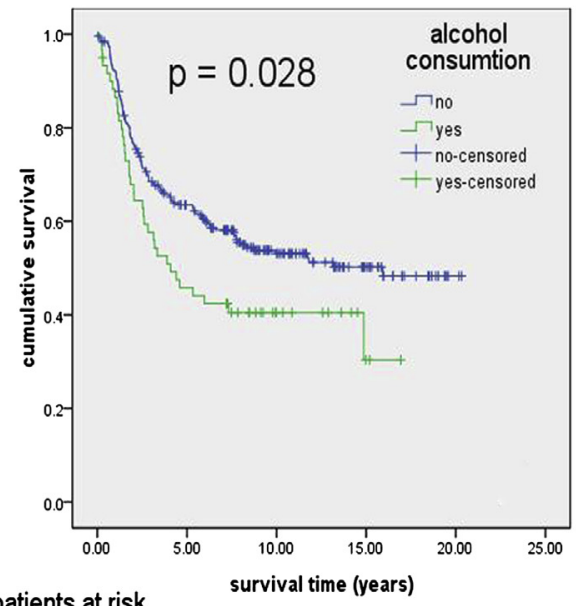

patients at risk

$\begin{array}{llllll}\text { no } & 246 & 120.5 & 56.5 & 19 & 1\end{array}$

B yes $\begin{array}{lllll}59.5 & 20.5 & 7 & 1\end{array}$

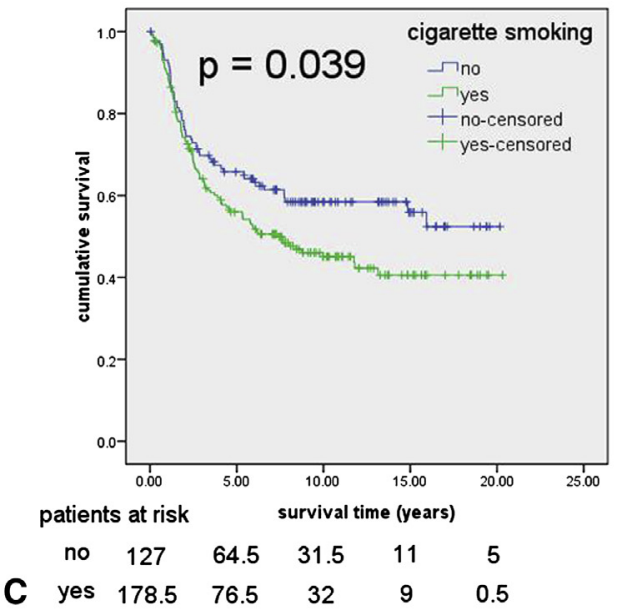

FIGURE 2. Kaplan-Meier survival curves for patients stratified by age (A), alcohol consumption (B), and cigarette smoking (C). Survival differences were analyzed using Cox regression analysis. 

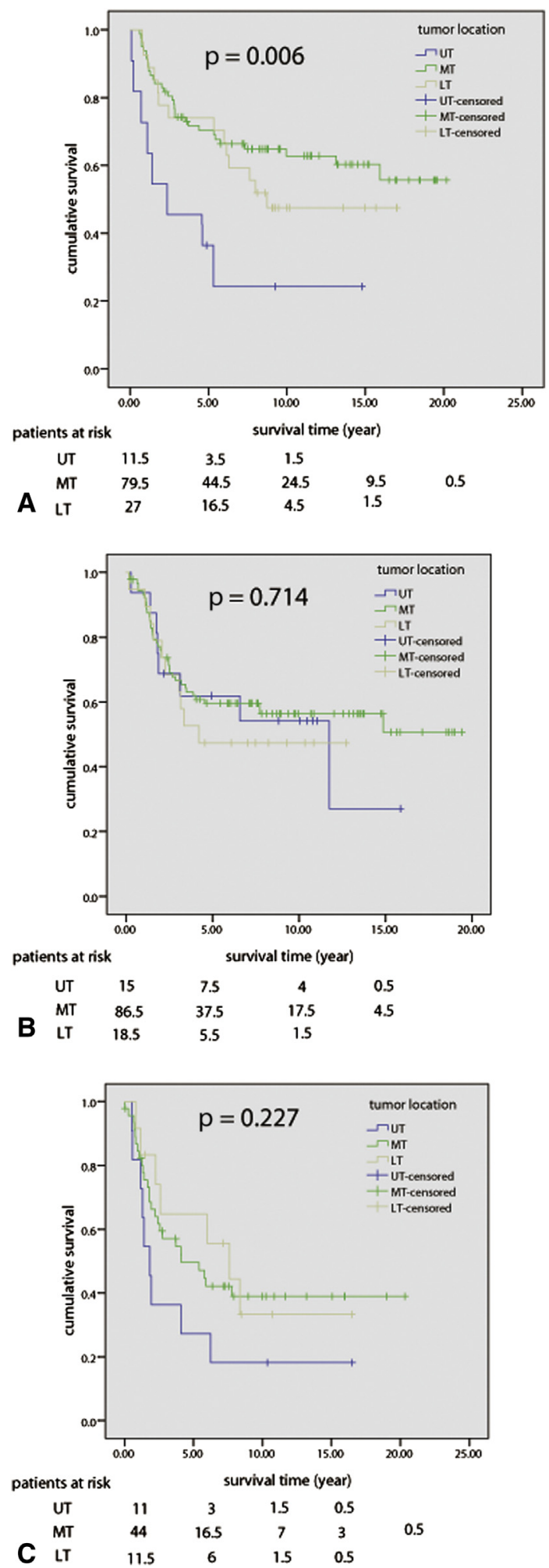

FIGURE 4. Kaplan-Meier survival curves for patients with well (A), moderately (B), and poorly (C) differentiated tumor stratified by tumor location. UT, Upper thoracic; $M T$, middle thoracic; $L T$, lower thoracic. located in the upper chest. In this regard, tumor location is probably less important with T2NOM0 tumors.

However, none of these studies focused solely on patients with pT2NOM0 ESCC, the main group affected by the new $\mathrm{G}$ stage classification. In our study, we found that tumor grade was an independent prognostic factor, and a relatively poorer differentiation was associated with poor survival. Different tumor locations were associated with survival, but they may not be independent prognostic factors. This may be attributed to the impaired accuracy of tumor location during the conversion from the sixth ${ }^{5}$ to the seventh edition $^{4}$ of the AJCC Cancer Staging Manual.

\section{CONCLUSIONS}

In addition to well-characterized factors affecting survival of patients with esophageal carcinoma, we showed that alcohol consumption and tumor grade were important prognostic factors for patient survival. Tumor grade was a strong prognostic factor in patient survival, as indicated in the seventh edition of the AJCC Cancer Staging Man$u a l,{ }^{4}$ whereas tumor location was not. The revised TNM staging system does not suggest a more efficient prediction of prognosis than the previous edition for patients with pT2NOMO ESCC.

\section{References}

1. Lin CS, Chang SC, Wei YH, Chou TY, Wu YC, Lin HC, et al. Prognostic variables in thoracic esophageal squamous cell carcinoma. Ann Thorac Surg. 2009; 87:1056-65.

2. Li H, Zhang Q, Xu L, Chen Y, Wei Y, Zhou G. Factors predictive of prognosis after esophagectomy for squamous cell cancer. J Thorac Cardiovasc Surg. 2009; $137: 55-9$.

3. Wong J. Esophageal resection for cancer: the rationale of current practice. Am J Surg. 1987;153:18-24.

4. Edge SB, Byrd DR, Compton CC, Fritz AG, Greene FL, Trotti A. AJCC Cancer Staging Manual. 7th ed. New York: Springer; 2010.

5. Greene FL, Page DL, Fleming ID, Fritz A, Balch CM, Haller DG, et al. AJCC Cancer Staging Manual. 6th ed. New York: Springer-Verlag; 2002.

6. Killinger WA Jr, Rice TW, Adelstein DJ, Medendorp SV, Zuccaro G, Kirby TJ, et al. stage II esophageal carcinoma: the significance of T and N. J Thorac Cardiovasc Surg. 1996;111:935-40.

7. Tachibana M, Kinugasa S, Dhar DK, Tabara H, Masunaga R, Kotoh T, et al. Prognostic factors in $\mathrm{T} 1$ and $\mathrm{T} 2$ squamous cell carcinoma of the thoracic esophagus. Arch Surg. 1999;134:50-4.

8. Rice TW, Mason DP, Murthy SC, Zuccaro G Jr, Adelstein DJ, Rybicki LA, et al. T2N0M0 esophageal cancer. J Thorac Cardiovasc Surg. 2007;133:317-24.

9. Elsayed H, Whittle I, McShane J, Howes N, Hartley M, Shackcloth M, et al. The influence of age on mortality and survival in patients undergoing oesophagogastrectomies. A seven-year experience in a tertiary center. Interact Cardiovasc Thorac Surg. 2010;11:65-9.

10. Eloubeidi MA, Desmond R, Arguedas MR, Reed CE, Wilcox CM. Prognostic factors for the survival of patients with esophageal carcinoma in the U.S.: the importance of tumor length and lymph node status. Cancer. 2002;95:1434-43.

11. Kountourakis P, Correa AM, Hofstetter WL, Lee JH, Bhutani MS, Rice DC, et al. Combined modality therapy of cT2N0M0 esophageal cancer: the University of Texas M. D. Anderson Cancer Center experience. Cancer. 2011;117:925-30.

12. Sundelof M, Lagergren J, Ye W. Patient demographics and lifestyle factors influencing long-term survival of oesophageal cancer and gastric cardia cancer in a nationwide study in Sweden. Eur J Cancer. 2008;44:1566-71.

13. Enzinger PC, Mayer RJ. Esophageal cancer. $N$ Engl J Med. 2003;349:2241-52.

14. Lee CH, Lee JM, Wu DC, Hsu HK, Kao EL, Huang HL, et al. Independent and combined effects of alcohol intake, tobacco smoking and betel quid chewing on the risk of esophageal cancer in Taiwan. Int J Cancer. 2005;113:475-82. 
15. Gao YT, McLaughlin JK, Blot WJ, Ji BT, Benichou J, Dai Q, et al. Risk factors for esophageal cancer in Shanghai, China. I. Role of cigarette smoking and alcohol drinking. Int J Cancer. 1994;58:192-6.

16. Khan NA, Teli MA, Mohib-Ul Haq M, Bhat GM, Lone MM, Afroz F. A survey of risk factors in carcinoma esophagus in the valley of Kashmir, Northern India. J Cancer Res Ther. 2011;7:15-8.

17. Shitara K, Matsuo K, Hatooka S, Ura T, Takahari D, Yokota T, et al. Heavy smoking history interacts with chemoradiotherapy for esophageal cancer prognosis: a retrospective study. Cancer Sci. 2010;101:1001-6.

18. Dickson GH, Singh KK, Escofet X, Kelley K. Validation of a modified GTNM classification in peri-junctional oesophago-gastric carcinoma and its use as a prognostic indicator. Eur J Surg Oncol. 2001;27:641-4.

19. Bogoevski D, Onken F, Koenig A, Kaifi JT, Schurr P, Sauter G, et al. Is it time for a new TNM classification in esophageal carcinoma? Ann Surg. 2008;247:633-41.
20. Hsu PK, Wu YC, Chou TY, Huang CS, Hsu WH. Comparison of the 6th and 7th editions of the American Joint Committee on Cancer tumor-node-metastasis staging system in patients with resected esophageal carcinoma. Ann Thorac Surg. 2010;89:1024-31.

21. Rice TW, Rusch VW, Apperson-Hansen C, Allen MS, Chen LQ, Hunter JG, et al. Worldwide esophageal cancer collaboration. Dis Esophagus. 2009;22: $1-8$.

22. Roder JD, Busch R, Stein HJ, Fink U, Siewert JR. Ratio of invaded to removed lymph nodes as a predictor of survival in squamous cell carcinoma of the esophagus. Br J Surg. 1994;81:410-3.

23. Doki Y, Ishikawa O, Takachi K, Miyashiro I, Sasaki Y, Ohigashi H, et al. Association of the primary tumor location with the site of tumor recurrence after curative resection of thoracic esophageal carcinoma. World J Surg. 2005;29: 700-7. 